\title{
GESNERUS
}

Vierteljahrsschrift für Geschichte der Medizin und der

Naturwissenschaften

Revue trimestrielle d'histoire de la médecine

Jahrgang - Volume - $1 \quad$ 1944 Heft - Fasc. - 3

\section{Der Rebbau im antiken Griechenland}

nach Theophrast, Causae plantarum, Kap. 11-16

Wohl der älteste und ausführlichste Bericht über den Rebbau im antiken Griechenland findet sich in dem Sammelwerk, das unter dem Namen $\pi \varepsilon \varrho i \varphi v \tau \tilde{\omega} \nu \alpha i \tau \iota \tilde{\omega} \nu$ «Über die Erklärung der Lebens-Erscheinungen der Pflanzen» überliefert ist und verschiedene z. T. unabhängig von einander verfaßte Abhandlungen des Theophrast von Eresos (370-285 a. Chr.) enthält. Sie sind im ersten vorchristlichen Jahrhundert durch Andronikos von Rhodos zu dem größeren, aber z. T. heterogenen Werk mit dem erwähnten Titel vereinigt worden.

\section{A. Allgemeines über Theophrasts Darstellung}

In den Kapiteln 11-16 des III. Buches dieses Werkes gibt Theophrast eine Darstellung der Verfahren, welche die Rebbauern durchzuführen pflegen, um gesunde Pflanzen mit möglichst gutem und reichlichem Ertrag zu erzielen. Seine Darstellung gliedert sich in folgende Abschnitte:

I. Rebensorten und Bodenarten, inwiefern sie zueinander passen. Kap. $11 \S 1-4$.

II. Die Vermehrung der Rebe durch Stecklinge. Kap. $11 \S 5$

Kap. 13.

1. Die Qualität der Stecklinge. Kap. $11 \S 5$.

2. Die geeigneten Böden.

3. Die Jahreszeit, in welcher die Stecklinge zu machen sind. $\$ 6$.

4. Die Vorbereitung des Bodens für die Aufnahme der Stecklinge.

5. Das Schneiden der Stecklinge. Kap. 12. Kap. 13. 
III. Die Behandlung der erwachsenen Reben. Kap. $14 \$ 1$ - Kap. 16

1. Der Rebschnitt.

Kap. 14.

a) $\mathrm{Das} \mathrm{MaB}$ des Schneidens. $\quad \S 3-8$.

b) Die Jahreszeit, in welcher geschnitten werden soll. Kap. 15.

2. Das Entfernen der sterilen Schosse. Kap. $16 \$ 1-2$.

3. Das Auseinanderlegen der Schosse und das Zurückschneiden des Stammes.

Kap. $16 \S 3$ a.

IV. Das Bestreuen der Trauben mit mineralischem Staub. Kap. 16 $\S 3 \mathrm{~b}-\S 4$.

Diese Ủbersicht zeigt zunächst, daß Theophrast seinen Stoff klar «u gliedern verstand. Dieser Stoff setzt sich aus zwei Bestandteilen zusammen, nämlich zunächst aus Angaben, welche Theophrast auf Grund eigener Beobachtung und Erfahrung gesammelt hat. Da er in Stagira auf der Halbinsel Chalkidike ein Landgut besaß, auf welchem er wohl auch Reben kultivierte, liegt die Annahme nahe, daß er diese Beobachtungen an seinen eigenen Rebbergen gemacht hat. Der zweite Bestandteil seiner Ausführungen wird durch Zitate anderer Rebenzüchter gebildet. Da diese nicht ganz einen Drittel der Abhandlung, nämlich 83 von 284 Teubner-Zeilen, einnehmen, handelt es sich bei dieser vorwiegend um Theophrasts eigene Erfahrungen und Anschauungen über die Rebkultur. Bei deren Beurteilung erfreute ich mich der fachmännischen Beratung des Herrn H. Faes, Directeur de la Station fédérale d'essais viticoles et arboricoles in Lausanne und des Herrn H. Schenk, Obergärtner des Botanischen Gartens in Bern. Für ihre wertvolle Hilfe spreche ich beiden Herren auch an dieser Stelle meinen verbindlichen Dank aus.

B. Der Inhalt von Theophrasts Schrift über den Rebbau

Im I. Hauptabschnitt über die Beziehungen, welche zwischen den Rebensorten und den Bodenarten bestehen, führt Theophrast aus, daß in einem festen und trockenen Boden diejenigen Rebensorten am besten gedeihen und am meisten Früchte tragen, deren einjährige Triebe fest sind, d.h. einen relativ dicken Holz-Rinden-Zylinder und wenig Mark haben. Das gelte für die weiß- wie für die rotfrüchtigen Reben. Allerdings hätten die rotfrüchtigen im allgemeinen einen dickeren Holz-Rinden-Zylinder, eigneten sich also besser für einen festen und trockenen Boden. $\mathrm{Zu}$ einem feuchten Boden paßten dagegen diejenigen Rebsorten am besten, in deren Schossen der Holzzylinder dünn und das Mark 
stark entwickelt ist. Der Charakter der Reben müsse also zum Charakter des Bodens passen. Da Theophrast diese Vorschriften in $\S 2$ damit begründet, daß die «festen» resp. dichten Reben das von ihnen aufgenommene Wasser im Sommer weniger rasch verlieren als die locker gebauten, handelt es sich bei letzteren offenbar um solche, die, wie wir jetzt sagen würden, hygrophilen Charakter haben, an trockenen Standorten zu viel Wasser abgeben und in Folge dessen leicht welken, während es sich bei den «festen» und dicht gebauten um Rassen von xerophilem Charakter handelt, welche sich für einen trockenen Boden eignen. So betrachtet, sind also Theophrasts Angaben durchaus verständlich; sie beruhen offenbar auf Erfahrung.

Seine Angabe, daß die rotfrüchtigen Reben auf einem festen und trockenen Boden im allgemeinen besser gedeihen und mehr Früchte tragen als in einem feuchten, wird mir von Herrn Kollegen Faes insofern bestätigt, als die Reben mit roten Trauben im allgemeinen in leichten und steinigen Böden gut gedeihen, während die Reben mit weißen Trauben kompaktere Böden vorziehen. Während diese Unterschiede in der Schweiz keinen großen Einfluß haben und darum bei der Rebkultur keine Rolle spielen, ist es sehr wohl denkbar, daß die Unterschiede zwischen trockenen und feuchten Böden in Griechenland größer sind als in unserm Lande, in welchem auch trockene Lagen mehr Regen erhalten, als dies in Griechenland der Fall ist.

Während also Theophrasts Angaben über die Beziehungen, welche zwischen verschiedenen Rebensorten und dem Wassergehalt des Bodens bestehen, offenbar auf Erfahrung beruhen, sind die physiologischen Erklärungen, die er für das Verhalten der Reben gibt, nicht stichhaltig. Wenn er z. B. in $\$ 4$ berichtet, daß die Rebe mehr Wasser brauche als andere Holzpflanzen, so beruht das offenbar auf der Überlegung, daß, weil die Rebe viel saftigere Früchte bildet als andere Bäume, sie auch mehr Wasser brauche als jene. Jedenfalls ist diese Behauptung nicht richtig. Behält doch die Rebe gerade wie der Ölbaum ihre Blätter auch in Zeiten großer Trockenheit länger als viele andere Holzpflanzen.

Ähnlich steht es mit Theophrasts Erklärung der Tatsache, daß die Rebsorten, welche ein großes Mark aufweisen, besonders viel Wasser brauchen, daß sie dank ihrem Besitz von zahlreichen und weiten Porenkanälen das aufgenommene Wasser rasch in die Höhe leiten und es in relativ großer Menge an die Luft abgeben. Infolge ihrer starken Wasseraufnahme seien solche Reben imstande, gute saftreiche Früchte zu 
bilden. Demgegenüber sollen die Sorten mit dickem Holz-Zylinder und engem Mark infolge der geringen Zahl ihrer Porenkanäle und deren kleinem Lumen nicht so viel Wasser in die Blätter schaffen. Da sie aber imstande sein sollen, das im Frühling aufgenommene Wasser in sich zurückzuhalten, könnten auch sie saftreiche Früchte bilden. Das ist reine Phantasie. Sie ist aber deshalb von Interesse, weil sie zeigt, auf welche Weise Theophrast in der Zeit, da er noch unter dem Einfluß seines Lehrers Aristoteles stand, die physiologischen Vorgänge der Pflanze mit Hilfe des $\lambda \dot{o} \gamma o \varsigma$, des verstandesmäßigen Denkens, zu erklären versuchte. Und da wir wissen, daß die das Wasser leitenden «Poren», d.h. die Gefäße, nicht im Mark, sondern im Holzkörper enthalten sind, demzufolge ein Stengel mit kräftig entwickeltem Holz das Wasser besser leitet als ein solcher mit großem Mark und schwachem Holzzylinder, fußt Theophrasts Erklärung nicht auf Beobachtung, sondern offenbar auf einer theoretischen Überlegung, und zwar auf der Poren- und Pneumatheorie des Philistion von Lokroi in Süditalien (um 388 a. Chr.), eines Arztes, der seine Theorie im Hinblick auf die katarrhalischen Erkrankungen unserer Respirationsorgane aufgestellt hat (vgl. Senn 1933 S. $58 \mathrm{f}$.). Wir werden dieser Theorie in der vorliegenden Schrift Theophrasts noch einmal begegnen (vgl. S. 81).

Theophrast teilt also in diesem ersten Hauptabschnitt zunächst die Erfahrungen mit, welche er selbst auf Grund von Beobachtungen gemacht hat. Während sich diese mit den Erfahrungen der heutigen Winzer in weitgehendem Maße decken, beruhen die Erklärungen, welche er für das Verhalten der Reben gibt, nicht auf Beobachtung, sondern auf naturphilosophischer Spekulation, welche die Pflanzenphysiologie als unzutreffend, ablehnen muß.

Im II. Hauptabschnitt, Kap. $11 \S 5-$ Kap. 13, bespricht Theophrast die Wahl und die Behandlung der Stecklinge, ${ }^{1}$ aus welchen junge Reben aufgezogen werden sollen. Von einigen Fachleuten werde empfohlen, für wasserreiche Böden dicke Stecklinge, für trockene und warme Böden dagegen Stecklinge von mittlerer Dicke zu wählen. Ferner hielten es diese Autoren für ratsam, in feuchten Böden bei den Reben, wie überhaupt bei allen Holzpflanzen, Markotten, also schon bewurzelte Ableger,

${ }^{1} \mathrm{Zu}$ Beginn dieses Abschnitts (Kap. 11 § 5) ist $\pi \varepsilon \varrho i \quad \delta \dot{\varepsilon} \tau \tilde{\omega} \nu \sigma \pi \varepsilon \varrho \mu c \dot{\tau} \omega \nu={ }^{\circ}$ Über die Samen, überliefert. Das ist aber offenbar unrichtig, da im ganzen Abschnitt von Samen nicht die Rede ist, wohl aber gleich nachher zwei Mal (in Sinnzeile 8 und 9) von $\varphi v \tau \varepsilon v \mu(i \tau \omega v$ $=$ Stecklingen. So ist auch an Stelle des überlieferten $\sigma \pi \varepsilon \varrho \mu a ́ t \omega \nu$ zu lesen. 
zu verwenden, weil diese weniger leicht faulen als gewöhnliche Stecklinge (Kap. $11 \S 5$ ). Bei der Angabe über den Zeitpunkt, in welchem die Stecklinge gepflanzt werden sollen, ob Mitte September oder erst Ende Dezember, handelt es sich um eine Frage, welche mit dem Charakter der griechischen Jahreszeiten eng zusammenhängt. Und da Theophrast für sie auch keine physiologische Erklärung gibt, beruhen diese Angaben offenbar auf alter Erfahrung und bedürfen darum keines Kommentars. Dasselbe gilt wohl auch für die weiteren Vorschriften, welche Theophrast für die Herstellung der Setzlöcher-ob weit oder eng-macht (Kap. 12 §1.) Wenn er ferner darauf hinweist, daß trockene und harte Böden wesentlich tiefer umgegraben werden müssen als weiche und feuchte, so deckt sich das nach gütiger Mitteilung des Herrn Kollegen Faes vollständig mit den Erfahrungen, welche man bei der Rebkultur im Wallis gemacht hat. Wenn aber Theophrast die Notwendigkeit des tiefen Umgrabens harter und trockener Böden damit erklärt, daß dadurch dem Boden viel Pneuma resp. Luft beigemischt werde, welche der Pflanze Nahrung biete, so spukt auch hier wieder Philistions Pneumatheorie, der wir schon im Abschnitt über die Eignung bestimmter Böden für bestimmte Rebensorten (S. 78) begegnet sind. Wenn dann ( $\$ 2$ Ende) Theophrast hinzusetzt, daß die Nahrung des Stecklings nicht allein aus der Erde, sondern auch von der Sonne und von der Luft stamme, so mutet uns das zunächst durchaus modern an, da ja die Pflanze aus der Luft die Kohlensäure erhält und sie mit Hilfe der Sonnenenergie reduziert. Wie aber aus dem Zusammenhang hervorgeht, stellte sich Theophrast vor, daß die Pflanzen die Luftnahrung mit den Wurzeln aus dem Boden aufnehmen. Daß in Wirklichkeit die Blätter das tun, konnte Theophrast noch nicht wissen. Immerhin ist es bemerkenswert, daß er wenigstens z. T. auf der richtigen Fährte gewesen ist.

Ferner gibt er an, daß man in Gegenden, welche starkem Wind ausgesetzt sind, die Stecklinge nicht in die Richtung gegen den Wind, sondern vom Winde weg einstecken solle. Dies beruht jedenfalls auf alter Erfahrung, da der Nordost-Wind, der im Aegäischen Meere während des Sommers vorherrscht (Struck, 1912, S. 36), sehr stark und dabei trocken ist und die jungen Triebe der Reben welken und verdorren macht.

Die Tatsache, daß Theophrast in diesem wie schon im I. Abschnitt für die Erklärung physiologischer Vorgänge in der Pflanze Philistions Pneuma- und Porentheorie zu Hilfe nimmt, beweist, daß er zur Zeit, 
da er die Schrift über den Rebbau verfaßte, noch ganz auf naturphilosophischem Boden stand. Diesen hat er aber im Alter völlig verlassen, sodaß seine Spätwerke, welche vorwiegend in seinen Historiae plantarum enthalten sind, rein naturwissenschaftlichen Charakter haben. Die Schrift über den Rebbau mit ihren naturphilosophischen Gedankengängen stammt somit, wie die Mehrzahl der übrigen in den Causae vereinigten Schriften, aus seiner Frühzeit (vgl. Senn 1933 S. 122 ff.). ${ }^{2}$

Im II. Unterabschnitt bespricht er die Behandlung, welche die Stecklinge nach ihrem Austreiben erfordern (Kap. 13 § 1-4). Er empfiehlt, die Triebe stark zurückzuschneiden, damit sie den Wurzeln nicht zu viel Nahrung entziehen. Auch sollen die Stecklinge, wie die alten Reben, unmittelbar vor dem Austreiben, d.h. im Frühling, geschnitten werden, weil dann der stehengelassene Trieb am besten ernährt werde. Von diesem im Frühling auszuführenden Schnitt, der die gute Entwicklung der vegetativen Sprosse zum Zwecke hat, unterscheidet Theophrast den zu Beginn des November vorzunehmenden Herbstschnitt, welcher den Fruchtansatz im folgenden Jahre bezweckt. Diese Maßnahmen erklärt er mit dem Verhalten des Saftstroms innerhalb der jungen Pflanze. Von Interesse ist die offenbar auf Grund alter Erfahrung gemachte Vorschrift, daß man die Setzlinge im frischen Boden früher, nämlich schon im dritten Jahre schneiden müsse, während die Setzlinge in einem schon vorher mit Reben bepflanzten Boden sich langsamer entwickeln, weil dieser schon etwas erschöpft sei. Diese Setzlinge müsse man darum erst später schneiden.

Auf alter Erfahrung beruhen offenbar auch die Ratschläge, welche Theophrast für die Formung der aus den Stecklingen gezogenen Reben gibt. Schließlich stellt er noch fest, daß der Strom der Nährstoffe in die embryonalen, in intensivstem Wachstum begriffenen Knospen gehe, eine Angabe, welche durchaus richtig ist. Zum Schluß wird betont, daß man bei allen diesen Maßnahmen nicht schematisch verfahren dürle, sondern auf die Qualität des Bodens, auf die Kräftigkeit der einzelnen

2 Das ergibt sich auch aus der Tatsache, daß Theophrast in dieser Darstellung der Rebkultur vier Male den Ausdruck aitia resp. aĩ $\iota \iota v$ «Ursache» anwendet (Kap. 14 §6, Kap. 15 §4 und 5, Kap. 16 §), einen Ausdruck, den er in seinen Spätschriften nur noch selten brauchte, weil dieser von den älteren Philosophen für die «Endursachen», also mit metaphysischer Bedeutung, verwendet worden war, deren Erkennbarkeit Theophrast später bestritt (vgl. Senn 1933 S.113). 
Triebe achten und beim Schneiden auch die vorherrschende Windrichtung berücksichtigen müsse.

So teilt dieser II. Hauptabschnitt über die Herstellung und die Behandlung der Stecklinge in der Hauptsache Erfahrungstatsachen mit, die allerdings bisweilen mit Hilfe von Theorien oder naturphilosophischen Prinzipien zu erklären versucht werden.

Im III. Hauptabschnitt (Kap. 14 $\$ 1-$ Kap. $16 \S 3$ z.T.), welcher die Hälfte der ganzen Darstellung einnimmt, befaßt sich Theophrast mit der Behandlung der ausgewachsenen Reben. Er unterscheidet dabei drei verschiedene Maßnahmen, nämlich:

1. Das Schneiden der verholzten Schosse,

2. Das Entfernen der sterilen jungen Schosse und

3. Das Auseinanderziehen der Schosse und das Zurückschneiden des Stammes.

Am eingehendsten bespricht er das Schneiden der Rebe und bezeichnet als Zweck dieser Maßnahme, die Rebe zu reichlicher Fruchtbildung zu veranlassen, ohne sie aber dadurch zu schwächen, sondern sie im Gegenteil zu befähigen, ein hohes Alter zu erreichen. Im ersten Unterabschnitt (Kap. 14 $\$ 3-8$ ) behandelt er das Maß des Schneidens und diskutiert die verschiedenen Ansichten anderer Autoren. Die einen richten sich beim Schneiden sowohl nach dem Charakter des Bodens als auch nach der Rebensorte, indem sie die auf warmem und trockenem Boden wachsenden Sorten, die ja nach dem I. Hauptabschnitt denselben Charakter wie der Boden haben sollen, stark zurückschneiden. Dadurch würden die Reben fruchtbarer, weil sie sich (wohl wegen der stärkeren Wurzelentwicklung) besser ernähren können. Dagegen müsse man auf feuchten und nährstoffreichen Böden die Schosse lang lassen, weil die Reben mit feuchtem Charakter an sich fruchtbarer seien und die Früchte an den distalen Enden der Schosse bilden.

Andere Autoren dagegen berücksichtigen beim Schneiden nur den Charakter der Rebe, nämlich ob diese ein großes oder ein kleines Mark habe. Es sind offenbar dieselben Autoren, deren Theorie Theophrast in bezug auf die Wahl des Bodens für die Rebkultur übernommen hat (vgl. S. 78). Sie behaupten, man müsse bei den Reben mit großem Mark viele kurze Sprosse stehen lassen, bei den Reben mit wenig Mark dagegen nur einige wenige, aber lange Sprosse. Auf diese Weise werde die Masse des Marks in beiden Fällen dieselbe, und dementsprechend auch die Fruchtbarkeit der Rebe. Diese Autoren begründen ihr Vorgehen 
damit, daß die Trauben aus dem Mark, die vegetativen Sprosse dagegen aus dem Holz-Rinden-Zylinder entstehen. Dieser Erklärung steht aber Theophrast mit Recht skeptisch gegenüber. Die hohe Einschätzung des Markes für die Blüten- und Fruchtbildung scheint übrigens auf einem Analogieschluß zu beruhen, indem ja auch dem Mark der Tierknochen im Altertum eine besonders große physiologische Bedeutung zugeschrieben wurde.

Der Vorschrift genannter Autoren, daß man, wenn die Rebe austreibt $(\$ 8)$, alle Triebe entfernen müsse, welche keine Blüten angelegt haben, pflichtet Theophrast mit Recht ohne Vorbehalt bei, ebenso, daß man den Trieben, welche Fruchtanlagen angesetzt haben, die

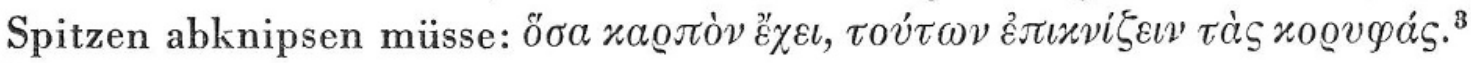

3 Bis dahin ist der Text völlig klar, nicht aber das darauf folgende: $\dot{\varepsilon} v$ aj̉ $\tau a \tilde{\zeta} \varsigma$

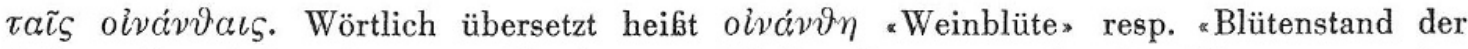
Rebe», der sich später zur Traube entwickelt. Dementsprechend müßten unter den Spitzen, welche in den Blütenständen abzuknipsen sind, die Ranken verstanden werden, welche gelegentlich aus den Blüten- und Fruchtständen der Rebe herauswachsen. Da aber dieser Fall relativ selten eintritt, und da durch die Entwicklung dieser Ranken der Traube nicht viel Nährstoffe entzogen würden, spielt die Entfernung dieser Ranken keine so große Rolle, daß sie neben dem Ausbrechen der sterilen Triebe und als diesem gleichwertig erwähnt werden müßte. Nun sagt aber der darauf folgende Satz, diese Manipulation werde ausgeführt, damit die Rebe das Wachstum nicht auf «diesen Zweig» $\tau o \tilde{v} \tau o$ cò $\varkappa \lambda \tilde{\eta} \mu a$ konzentriere. Hier ist somit wieder, wie im ersten Satzteil, von einem

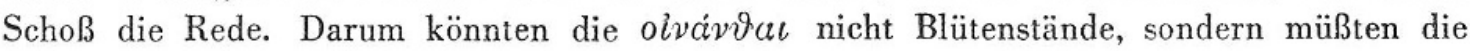
Schosse bedeuten, welche Blütenstände aufweisen. Diese Bedeutung hat aber das Wort sonst nicht. Nun kann die Schwierigkeit, welche der Oinanthe-Passus verursacht, dadurch behoben werden, daß man ihn zunächst von der überlieferten Stelle entfernt. Der Satz erhält dann den durchaus vernünftigen Sinn: «An diesen (nämlich den Sprossen, welche Fruchtanlagen erkennen lassen) muß man die Spitzen abkneifen, damit die Rebe das Wachstum nicht auf diese Sprosse konzentriere.

Als was ist aber der Oinanthe-Passus aufzufassen? Offenbar hat er sich ursprünglich

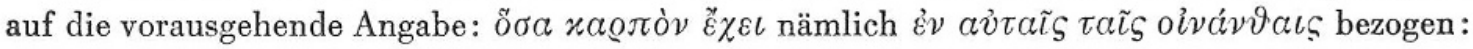
«Die Sprosse, welche Fruchtanlagen, und zwar gerade in den Blütenständen aufweisen». Diese Feststellung kommt uns jetzt selbstverständlich und darum überflüssig vor. Sie war es aber nicht, als der Passus niedergeschrieben wurde. Denn daß sich die Früchte stets aus Blüten entwickeln, war für Theophrast nicht selbstverständlich, weil z. B. der Feigenbaum Früchte reift, ohne, wie es wenigstens damals schien,

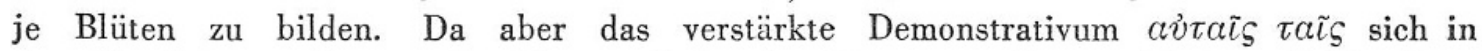
den überlieferten Text nicht fügt, und da der Passus an der unrichtigen Stelle überliefert ist, handelt es sich offenbar nicht um einen ursprünglichen Bestandteil des Textes, sondern um eine erklärende Bemerkung, die Theophrast oder ein anderer Autor nachträglich an den Rand geschrieben hat, und die erst nachträglich in den Text, in diesem aber an die unrichtige Stelle geraten ist. 
Das würde dem «Kappen» oder «Abkneifen» der heutigen Rebbauern entsprechen; letzterer Ausdruck stimmt übrigens mit dem Ė $\pi x v i \zeta \varepsilon v \nu$ auch sprachlich überein. Dabei werden 3-4 Knoten des Stengels mit je einem Blatt oberhalb des Blütenstandes stehen gelassen. Würde nämlich unmittelbar oberhalb von dessen Insertionsstelle geschnitten, so könnte sich die Traube wegen der Nähe der Wundstelle nicht richtig entwickeln. ${ }^{4}$ Übrigens behandelt Theophrast das Entfernen der sterilen, noch krautigen Triebe erst in Kap. $16 \S 1-2$ (vgl. S. 87 f).

Über die Jahreszeit, in welcher die Reben geschnitten werden müssen (Kap. 15), gehen die Ansichten der von Theophrast zitierten Autoren auseinander. Die einen richten sich ausschließlich nach dem Boden und schreiben vor, daß man die auf trockenen, warmen Böden stehenden Reben gleich nach dem Laubfall, also schon im Herbst, schneiden müsse, die auf regenreichem, kaltem Boden dagegen erst vor dem Austreiben, also im Frühling. Theophrast begründet diese Vorschrift mit ähnlichen Spekulationen über das Verhalten des aufgenommenen Wassers $(\S 1)$, welche er schon bei der Erklärung der Beziehungen zwischen Rebsorten und Boden (Kap. $11 \S 2$, vgl. S. 79) verwendet hat. Er weist dann auf die Gefahr hin, daß im Winter viele Knospen infolge der Kälte zu Grunde gehen $(\$ 2)$. Von Interesse ist auch die Feststellung der von Theophrast zitierten Autoren (Kap. $15 \$ 4){ }_{,}^{4}$ daß die auf feuchtem Boden stehenden, sowie die luxurierenden Reben stark zurückgeschnitten werden müßten, weil sie sonst nur vegetative Triebe bilden. Im Anschluß an diese Ausführungen behandelt $\S 3$ die Frage, in welchem Zeitpunkt die Rebe die Blüten-Anlagen für das folgende Jahr erkennen lasse. Dies sei, so behaupten einige Autoren, erst nach der Weinlese der Fall, weil die Rebe nicht zu gleicher Zeit ihre Früchte reifen und die Blüten-Anlagen für das kommende Jahr bilden könne. Diese Begründung geht offenbar auf einen Anhänger des Aristoteles zurück, da letzterer wiederholt darauf hingewiesen hat, daß die Natur nicht imstande sei, mehrere Dinge gleichzeitig durchzuführen. Obwohl ja auch Theophrast ein Schüler des Aristoteles gewesen ist, bestreitet er die Richtigkeit dieser Angabe und stellt mit Recht fest, daß die Blüten schon im Som-

${ }_{4}^{4}$ Der $\S 4$ des Kap. 15 ist nicht an der richtigen Stelle überliefert; führt er doch aus, weshalb man den Schnitt an den in warmem Boden stehenden Reben schon im Herbst, an den in feuchtem Boden stehenden dagegen erst im Frühling vornehmen müsse. Das wurde in $\S 1$ besprochen, während in $\S 2$ und 3 vom Einfluß des Bodens nicht mehr die Rede ist. Der $\$ 4 \mathrm{mu}$ darum zwischen die $\$ \S 1$ und 2 eingeschoben werden. 
mer angelegt werden, wenn die Rebe noch in vollem Saft steht. Aus letzterem werde nämlich die Traube gebildet, da er durch die Sonnenwärme eingedickt werde. Unter diesen Umständen entstehe eine große Zahl von Trauben. Obwohl uns diese rein physikalische Auffassung allzu primitiv erscheint, ist sie insofern richtig, als zwar nicht die Wärme, wohl aber das Licht der Sonne eine starke Assimilationstätigkeit und dementsprechend einen hohen Zuckergehalt der Pflanzen zur Folge hat, der seinerseits die Bildung von Blüten-Vegetationspunkten begünstigt.

Alle diese Vorschriften faßt dann Theophrast dahin zusammen, daß die Jahreszeit, in welcher geschnitten werden muß, und das Maß des Schneidens sich sowohl nach der Art des Bodens als auch nach den Sorten der Rebe richten müsse. Gebe es doch gewisse Sorten, welche trotz starkem Schneiden vegetativ bleiben $(\$ 5)$. Diese kurze Bemerkung ist neben der in Kap. $15 \$ 4$ enthaltenen Erwähnung der Behandlung der luxurierenden Reben die einzige Angabe, welche sich auf das Schneiden der verschiedenen Sorten von Reben bezieht. Damit soll offenbar der in $\$ 1$ enthaltenen Exposition Genüge getan werden, welche die Wahl der Jahreszeit für den Rebschnitt auch von der Sorte der Reben will abhängen lassen. Zum Schluß bemerkt Theophrast, daß die Reben, welche man wenig zurückschneide, zwar viel tragen, in Folge dessen aber früh alterten.

Wie mir Herr Kollege Faes mitteilte, nimmt man heute beim Rebschnitt wenig Rücksicht auf die Beschaffenheit des Bodens, in welchem die Reben stehen, wohl aber auf deren Sorte. Die einen bilden nämlich die Trauben aus denjenigen Knospen, welche unten an den Schossen stehen, während andere Sorten sie an deren obern Enden entwickeln. Auf die Wichtigkeit, die dieser Unterschied für den Rebschnitt hat, weist übrigens auch Theophrast in Kap. $14 \$ 3$ Ende und $\$ 7$ Ende hin. Ferner schneidet man bei uns die Reben nicht durchwegs im Herbst, weil eine geschnittene Rebe leichter erfriert (Mitteilung von Herrn H.Schenk). Wie wir S. 85 gesehen haben, machte schon Theophrast (Kap. 15 §) auf diese Gefahr aufmerksam.

Jedenfalls kann gesagt werden, daß der Abschnitt über das Schneiden der Rebe vorwiegend auf Erfahrung beruht, deren Tatsachen Theophrast allerdings mit physiologischen Hypothesen verständlich zu machen sucht, welche aber unsern Kenntnissen gegenüber nicht standhalten.

Während im eben behandelten Abschnitt fast ausschließlich vom Schneiden der holzigen einjährigen Triebe die Rede gewesen ist, handelt 
der nächste Unter-Abschnitt Kap. $16 \$ 1-2$ von der Entfernung bestimmter, diesjähriger, noch krautiger Triebe, von der $\beta \lambda a \sigma \tau o \lambda o \gamma i \alpha$. Dabei unterscheidet Theophrast

1. das Entfernen aller Triebe, die keine Blütenanlagen tragen. Das soll vorgenommen werden, sobald man erkennen kann, welche Triebe Blütenstände angelegt haben,

2. ein zweites Entfernen der Triebe, an welchen keine Blütenanlagen vorhanden sind, nachdem die Rebe ein zweitesmal gejätet worden ist, aber noch bevor sie blüht.

Vergleicht man diese Vorschrift mit den Vorkehrungen, welche unsere Winzer ausführen, so besteht insofern eine Übereinstimmung, als auch letztere unter zwei Malen junge Triebe entfernen. Dabei handelt es sich beim erstenmal, sobald zu erkennen ist, welche Triebe Blütenanlagen tragen, um die Entfernung der steril bleibenden Sprosse als Ganzes. Das nennt man das «Ausbrechen» oder «Auspflücken». Das zweite Mal dagegen werden heutzutage die Spitzen der fertilen Triebe abgeschnitten, was z. T. vor, z. T. nach der Blüte ausgeführt wird (Schmitthenner 1910, S. 59); man nennt dies das «Kappen». Außerdem werden dann auch die aus den Blattwinkeln herauswachsenden «Geiztriebe» auf $1-2$ Blätter zurückgeschnitten («Ausgeizen). Von diesen drei Manipulationen erwähnt Theophrast in Kap. 14 $\$ 8$ das Auspflücken und das Kappen, in Kap. $16 \S 1$ dagegen ein zweimaliges «Auspflücken» $\beta \lambda \alpha \sigma \tau 0 \lambda o \gamma i a$. Unter dem zweiten Auspflücken versteht er vermutlich das Ausgeizen und wohl auch die Entfernung von Trieben, welche sich seit dem ersten Ausbrechen entwickelt haben (Scheu 1936, S. 130).

Nach diesen Ausführungen über das Entfernen der sterilen Schosse stellt Theophrast fest, daß die Rebe bis Ende Juli, d.h. bis zum Aufgang des Hundsterns, treibe; dann stelle sie das Treiben ein, die Sprosse begännen zu verholzen und führten die Frucht der Reife entgegen. An schattigen und feuchten Standorten halte das Sprossen länger an. Theophrast erklärt dies damit, daß die Rebe die daselbst schwächer wirkende Wärme der Sonne «überwinde». In Wirklichkeit hält aber das vegetative Wachstum deshalb dort länger an, weil die Intensität des Lichts, das ja auf das Wachstum der Pflanzen durchwegs hemmend wirkt, dort nicht so stark ist, wie an sonnigen Standorten.

Mit einigen Bemerkungen über das Auseinanderlegen der Schosse und über das Zurückschneiden des Stamms (Kap. 16§3, Z. 1-2) schließt Theophrast den Abschnitt. Wie wir sahen, berichtet er darin aus- 
schließlich über Erfahrungen der Rebbauern und versucht sie bisweilen mit physiologischen Hypothesen zu erklären, ohne jedoch naturphilosophische Theorien zu verwenden.

Im IV. Hauptabschnitt (Kap. $16 \S 3, \mathrm{Z} .2-\$ 4$ ) bespricht er das Bestreuen der Trauben mit mineralischem Staub. Dabei vertritt er die Ansicht, daß diese Prozedur erst an den reifen Trauben ausgeführt werden soll und nicht, wie andere Autoren behaupten, schon an den unreifen. Theophrast begründet seine Auffassung damit, daß der Staub das Ausreifen hemme, indem er den Drang der Pflanze zum Reifen in andere Bahnen lenke. Darum hielten einige Autoren diese Maßnahme überhaupt für schädlich. Theophrast ist nicht dieser Ansicht. Daß sie den Trauben förderlich sei, ergebe sich einerseits aus dem Umstand, daß das Überstäuben tatsächlich im Brauche sei: $\dot{\eta} \chi \varrho \varepsilon i a \mu a \varrho \tau v \varrho \varepsilon \tilde{\iota}$, andrerseits auch aus den bei andern Pflanzen damit gemachten Erfahrungen. So würden die Gurken zarter, wenn man sie, wie dies bei den Megarern üblich sei, mit Staub bedecke. Dieser Effekt werde deshalb erzielt, weil die Sonne die Gurken austrockne und sie holzig werden lasse, während sie durch die Bedeckung mit Staub oder mit den Blättern vor Verholzung geschützt würden, weil sie sich dann in feuchter Luft entwickelten. Während diese Überlegung wenigstens teilweise der Wirklichkeit entsprechen dürfte, müssen Theophrast's Mutmaßungen über die Wirkungsweise des mineralischen Staubes auf die Trauben als unhaltbar bezeichnet werden. Ist doch weder an eine direkte oder indirekte Ernährung der Trauben durch den Staub, noch an einen durch letzteren ausgeübten Schutz gegen die Sonne zu denken, was Theophrast beides für die Erklärung des Zartwerdens der Gurke in Betracht zieht.

Da aber im Gegensatz zu den Gurken bei der Rebe eine Verholzung der Beeren nicht in Frage kommt, scheint die Idee, daß ihre Bedeckung mit Staub nützlich sei, ursprünglich auf der Analogie mit einem andern Vorgang zu beruhen. Wie nämlich Theophrast in Kap. $18 \S 1$ desselben Buches der Causae treffend ausführt, müssen die weiblichen Dattelpalmen mit dem Pollen der männlichen Bäume bestäubt werden, damit die Fruchtanlagen nicht abfallen, sondern sich zu Datteln entwickeln. Daß es sich dabei um einen Geschlechtsakt handle, hat Theophrast zwar in Betracht gezogen, aber nicht als eine Tatsache anerkannt. Da er nun für den Blütenstaub der Dattelpalme dasselbe Wort wie für den mineralischen Staub, nämlich xovı@ er jedem beliebigen Staub einen günstigen Einfluß auf die Früchte 
beigemessen zu haben. Jedenfalls wird das Bestäuben der Trauben, wenigstens in Mittel-Europa, weil zwecklos, nicht ausgeführt.

\section{Die Bestandteile der Abhandlung über den Rebbau.}

Die Analyse von Theophrast's Abhandlung über den Rebbau ergibt somit, daß in ihr neben seinen eigenen Angaben auch Zitate anderer Autoren enthalten sind, welche er z. T. als richtig anerkennt, z. T. aber auch als unzutreffend ablehnt. Unter diesen Autoren lassen sich mindestens drei verschiedene Persönlichkeiten oder Gruppen von solchen unterscheiden, nämlich:

a) solche, welche ausschließlich die Konsistenz (hart oder weich) und den Wassergehalt der Böden berücksichtigen (Kap. $15 \S 1$.),

b) solche, welche ausschließlich die Konstitution der Rebensorten, speziell im Hinblick auf die Größe ihres Markes in Betracht ziehen (Kap. 14 \$ 4-6),

c) solche, welche sowohl die Bodenarten als auch die Rebensorten berücksichtigen (Kap. 14 §3),

d) solche, welche sich nicht in diesen Gruppen unterbringen lassen, vielleicht aber doch zu ihnen gehören (Kap. 14 §7-8, Kap. $15 \S 3$ und Kap. $16 \S 3)$.

Unter den Angaben, welche Theophrast selbst vertritt, sind ebenfalls verschiedene Gruppen zu unterscheiden, nämlich:

a) Angaben von Tatsachen und Ansichten,

a) die er von den vorhin erwähnten Autoren übernommen hat, so z. B. die Wichtigkeit des Massenverhältnisses vom Mark einerseits, und vom Holz-Rinden-Zylinder anderseits bei der Auswahl der Rebensorten für bestimmte Böden (Kap. $11 \S 1$ ),

$\beta$ ) die er als seine eigenen Ansichten angibt und welche in den Zitaten der andern Autoren nicht enthalten sind, sondern offenbar auf seinen Erfahrungen als Weinbergbesitzer beruhen, so z. B. daß gewisse Rebensorten trotz starkem Schneiden unfruchtbar bleiben, und daß wenig zurückgeschnittene Reben viel tragen, aber früh alt werden (Kap. $15 \$ 5$ ), sowie die Angaben über das Wachstum der Rebe, die Einstellung des Wachstums und die darauf folgende Verholzung der Triebe (Kap. $16 \S 2$ ).

b) Erklärungen und Begründungen. 
a) mit Hilfe eigener physiologischer Hypothesen, z. B. über die Wasseraufnahme und -abgabe der Rebe (Kap.11 $\$ 2$ und Kap. $15 \S 1$ ), Bildung der Trauben infolge der Eindickung des Rebsaftes (Kap. $15 \S 3$ ), Ernährung der Trauben durch den Staub (Kap. $16 \$ 4$ ).

B) mit Hilfe naturphilosophischer Auffassungen früherer Philosophen, so der Pneuma- und Poren-Theorie des Philistion von Lokroi (Kap. 11 $\S 2$ und Kap. $12 \S 2)$.

c) Kritiken an den Ansichten anderer Autoren: Entstehung der Früchte aus dem Mark (Kap. 14 \$6-7).

Soweit die historische Analyse.

\section{Die Beziehungen zwischen der antiken und der heutigen Rebkultur.}

Obwohl es unhistorisch wäre, die Ansichten der Rebbauern des antiken Griechenlands vom Standpunkt der jetzigen Lehre über den Rebbau zu beurteilen, ist es immerhin interessant festzustellen, welche Ansichten der alten Griechen im Laufe der seit der Abfassung von Theophrasts Schrift verflossenen 2200 Jahre ihre Geltung bewahrt haben. So z. B. die Regel, daß man trockene und harte Böden für die Rebkultur tiefer umgraben muß als feuchte und feinkörnige Böden, ebenso daß die Reben, welche Rotweine liefern, im allgemeinen in einem leichten und locker-steinigen Boden besonders gut gedeihen, während die den Weißwein liefernden Reben eher kompakte, schwere Böden bevorzugen. Der Rebschnitt wird heutigentags hauptsächlich auf Grund der Rebensorten ausgeführt, besonders mit Rücksicht auf die Stelle, an welcher die fruchtbaren Triebe entstehen, und nur in speziellen Fällen mit Rücksicht auf die Qualität der Böden, also wenigstens z. T. auch noch entsprechend den im Altertum geltenden Regeln. Endlich werden auch jetzt noch, wie zu Theophrast's Zeiten, die sterilen Schosse ganz ausgebrochen, während an den fertilen die Spitze «abgeknipst» wird.

Aus dem Vergleich der von Theophrast erwähnten Maßnahmen der Rebkultur mit den heute noch im Gebrauch stehenden geht somit klar hervor, daß die Rebe im Zeitraum von 2200 Jahren ihre Eigenschaften nicht verändert hat, und daß die Änderungen, welche seither in die Methoden ihrer Kultur eingeführt worden sind, ausschließlich auf den Fortschritten der Kenntnis der physiologischen Eigenschaften der Rebe beruhen. Und da alle im Vorstehenden behandelten Vorkehrungen, welche sich seit Theophrasts Zeiten bis heute erhalten haben, auf Be- 
obachtung und Erfahrung beruhen, zeigt es sich einmal mehr, daß Beobachtung und Erfahrung in der Wissenschaft und in der Praxis unbegrenzten Bestand haben, während den theoretischen Ansichten häufig nur ein ephemeres Leben beschieden ist.

\section{G. Senn.}

\section{Literatur}

1936 Scheu, Georg, Mein Winzerbuch, Reichsnährstand-Verlags-G. m. b. H. Berlin N. 4.

1910 Schmitthenner, F., Weinbau und Weinbereitung. Aus Natur und Geisteswelt, 332. Bändchen, B. G. Teubner, Leipzig.

J.933 Senn, G., Die Entwicklung der biologischen Forschungsmethode in der Antike und ihre grundsätzliche Förderung durch Theophrast von Eresos. VIII. Band der Veröffentlichungen d. Schweiz. Ges. f. Geschichte d. Medizin u. d. Naturwissenschaften, H. R. Sauerländer \& Co., Aarau u. Leipzig.

1912 Struck, Adolf, Zur Landeskunde von Griechenland, Kulturgeschichtliches und Wirtschaftliches. IV. Serie, 4. Heft der «Angewandten Geographie». H. Keller, Frankfurt a. M.

\section{Einiges aus der Praxis von Galen}

Wenn man sich mit den Ärzten der Antike beschäftigt, so kann man diese Aufgabe von einem doppelten Standpunkt aus in Angriff nehmen. Entweder forscht man ihren medizinischen Gedankengängen nach, untersucht, was für neue Hypothesen sie aufgestellt, auf wessen Fußstapfen sie sich weiterentwickelt haben und dergleichen mehr. Oder man beschränkt sich lediglich darauf, sich nur ein anschauliches Bild von ihrer medizinischen Tätigkeit zu machen, m. a. W. sie mehr persönlich zu erfassen, um sich dadurch eine Vorstellung von ihrer ärztlichen Tätigkeit zu verschaffen. Diese letztere Betrachtungweise soll in dieser Arbeit bezüglich der Gestalt Galen's die wegleitende sein.

Gebürtiger Kleinasiate - wahrscheinlich geboren im Jahre 129 n. Chr. - hatte er nach mehrjährigem medizinischem Studium in Smyrna, Korinth und Alexandria als 28jähriger Arzt sich in Pergamon niedergelassen, wo er von dem amtierenden Oberpriester zum alleinigen Gladiatorenarzt ernannt wurde. Interessant ist $\mathrm{es,} \mathrm{da}$ ß er gerade die ältesten Schulen, speziell Alexandria, für seine Bildung aufsuchte, wo im 4. und 3. vorchristlichen Jahrhundert unter Herophilos und Erasistratos ein neues medizinisches Leben aufblühte, das sich mit einer Umstellung der bisherigen anatomischen und physiologischen Probleme beschäftigte. Vor einigen Jahren ist es gelungen, die Stätte von Galen's damaliger Tätigkeit, 